\title{
Coding packets over reordering channels
}

\author{
Douglas J. Leith and Dinkar Vasudevan \\ Hamilton Institute, National University of Ireland, Maynooth
}

\begin{abstract}
We define the "reordering channel" which approximates packet communication over multipath networks. We consider deterministic packet arrivals with finite horizon decoding of individual packets and analyze the error probability for a two stage coding scheme.
\end{abstract}

\section{INTRODUCTION}

We define a class of channels that we call the "reordering channel", and consider packet communication over channels belonging to this class. In this channel, time is slotted and with probability $p_{j}$, a packet sent at timeslot $i$ arrives before slot $i+j$ at the receiver. The delay of each packet is assumed to be independent. Thus, in this channel, packets could be received in an order which differs from the order of arrival of the packets at the sender. We define this channel with the objective of approximating packet communication over multipath networks. A special case that we will consider closely is when $p_{j}$ is constant for all $j$, namely the channel is an erasure channel.

We assume that packets arrive at rate $\lambda$ at the sender. Each packet has a deadline of $n$ slots, by which time it must be decoded at the receiver. Such a constraint is natural in applications such as video streaming. Figure 1 illustrates the problem setup. A packet is in error if the receiver fails

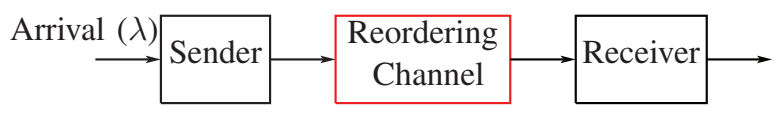

Fig. 1. Problem Setup

to decode the packet by the deadline. We are interested in encoding packets with the objective of minimizing the fraction of packets in error (the error probability). To this end, we propose a two-stage coding scheme and analyze how the error probability trades off with the decoding deadline $n$ for our scheme.

We emphasize here that traditional block codes are ill-suited for the problem due to the per-packet deadline constraints. The reason is that block coding is done on large chunks of packets, thus there is a delay incurred in buffering the required number of packets before encoding as well as in waiting for the requisite number of packets before decoding. The solution lies in constructing streaming causal codes which prioritize the delivery of earlier data packets. Such codes have been proposed and analyzed in [1] and [2] in the context of packet erasure channels and discrete memoryless channels respectively. Our main contribution is our proposed coding scheme, that we analyze for the class of reordering channels.
We derive bounds to the exponent of error probability of our coding scheme as a function of the decoding deadline $n$.

The paper is organized as follows: In Section II, we describe the coding scheme and setup the optimization problem corresponding to the scheme. In Section III, we analyze the error probability when the arrivals are deterministic. In Section IV, we show simulation results for the error probability versus the decoding delay $n$ for the reordering channel as well as the erasure channel. Apart from validating the bounds, we also show how our scheme compares with a causal Fountain coding scheme, and demonstrate the benefit of using our scheme.

\section{Coding SCHEME AND SUM LOG UTILITY}

The encoding has two stages: The first stage is the encoding of the individual packets using a standard generator matrix code such as a Reed-Solomon code or a Fountain code. If each data packet has $N$ bits, we will assume that the code is such that the packet can be reconstructed from any $N$ encoded bits. The second stage allocates the content of the encoded packets of the first stage across the transmitted packets. This is illustrated in Figure 2 for the case when $n=3$ and $\lambda=1$ (packets arrive in every timeslot). In this example, the bits in packet $C(1)$ are allocated over three consecutive timeslots since there is no point allocating the bits to packets that are sent later than the decoding deadline. In general, the content of the encoded packet $C(i)$ is allocated across packets sent in the time intervals $[i, i+n-1]$. The allocation is done under a constraint on the size of transmitted packets. In our example, the constraint is that $C(i)_{1}+C(i-1)_{2}+C(i-2)_{3}$ is constant across $i$. The optimal allocation is one that minimizes

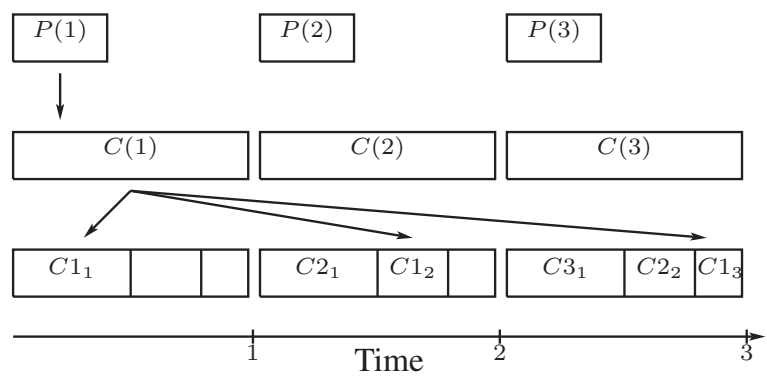

Fig. 2. Two stage encoding: $P(1)$ is encoded to $C(1)$, the contents of which are allocated across subpackets $C(1)_{1}, C(1)_{2}, C(1)_{3}$ across 3 timeslots.

the sum log of error probabilities across time slots. In the case of deterministic arrivals, we will see that this objective is equivalent to the error probability. Let $N_{i}(j)$ represent the number of encoded bits of data packet $i$ (i.e., bits of $C(i)$ ) in the packet sent at time $j$. We expect that a good code will 
allocate the encoded bits so that $N_{i}(i) \geq N_{i}(i+1) \cdots \geq$ $N_{i}(i+n-1)$. This is because packets sent earlier have a greater chance of arrival within the deadline. The objective we minimize is given by

$$
\sum_{i \in \mathcal{A}} \log \operatorname{Pr}\left(\sum_{l=i}^{i+n-1} N_{i}(l) \delta_{i, l}<N\right)
$$

where $\mathcal{A} \subseteq \mathbb{Z}$ denotes the set of the slot indexes where a data packet arrives and $\delta_{i, l}$ is the indicator of the packet sent at time $l$ reaching by the (deadline) time $n+i$. The $\operatorname{Pr}\left(\delta_{i, l}=1\right)=$ $p_{i+n-l}$, however for ease of notation we will define $p_{i, l} \triangleq$ $\operatorname{Pr}\left(\delta_{i, l}=1\right)$. Note the summation inside the probability term is over packets sent in the interval $[i, i+n-1]$. The implicit assumption is that the packet sent at time $i+n$ does not reach by time $i+n$, thus $p_{0}=0$. The probability expression in (1), being combinatorial, is difficult to evaluate and we therefore upper bound the objective (using the Chernoff bound) as

$$
\min _{\theta \geq 0} \sum_{i \in \mathcal{A}} \log \mathbb{E}\left[e^{-\theta_{i}\left(\sum_{l=i}^{i+n-1} N_{i}(l) \delta_{i, l}-N\right)}\right]
$$

where $\theta$ denotes the vector of $\theta_{i}$ 's. In the following, we determine the allocation $\left\{N_{i}(j)\right\}_{i}$ that minimizes the bound to the error probability. The objective is simplified as

$$
\begin{aligned}
\min _{\theta \geq 0} & \sum_{i \in \mathcal{A}} \log \mathbb{E}\left[e^{-\theta_{i}\left(\sum_{l=i}^{i+n-1} N_{i}(l) \delta_{i, l}-N\right)}\right] \\
& =\min _{\theta \geq 0} \sum_{i \in \mathcal{A}}\left(\theta_{i} N+\log \mathbb{E}\left[e^{-\theta_{i} \sum_{l=i}^{i+n-1} N_{i}(l) \delta_{i, l}}\right]\right) \\
& \stackrel{(a)}{=} \min _{\theta \geq 0} \sum_{i \in \mathcal{A}}\left(\theta_{i} N+\sum_{l=i}^{i+n-1} \log \mathbb{E}\left[e^{-\theta_{i} N_{i}(l) \delta_{i, l}}\right]\right) \\
& =\min _{\theta \geq 0} \sum_{i \in \mathcal{A}}\left(\theta_{i} N+\sum_{l=i}^{i+n-1} \log \left(1-p_{i, l}+p_{i, l} e^{-\theta_{i} N_{i}(l)}\right)\right)
\end{aligned}
$$

The equality (a) follows from the independence of the event of the sent packet at time $l$ arriving by time $i+n$. The objective has to be minimized with respect to the $\theta_{i}$ 's and the allocations $N_{i}(j)$ subject to the constraint on the size of the sent packet, $\sum_{i \in \mathcal{A} \cap\{j-(n-1), \ldots, j\}} N_{i}(j)=N / R$. It is assumed that $1 / R$ is the ratio of the size of a transmitted packet to a data packet. There are also non-negativity constraints $\theta_{i} \geq 0$ and $N_{i}(j) \geq$ 0 . The Lagrangian for the optimization problem is

$$
\begin{aligned}
& \sum_{i \in \mathcal{A}}\left(\theta_{i} N+\sum_{l=i}^{i+n-1} \log \left(1-p_{i, l}+p_{i, l} e^{-\theta_{i} N_{i}(l)}\right)\right) \\
& +\sum_{l \in \mathbb{Z}} \gamma_{l}\left(\sum_{i \in \mathcal{A} \cap\{l-(n-1), \ldots, l\}} N_{i}(l)-N / R\right) \\
& -\sum_{i \in \mathcal{A}, l \in \mathbb{Z}} \lambda_{i, l} N_{i}(l)-\sum_{i \in \mathcal{A}} \mu_{i} \theta_{i} .
\end{aligned}
$$

The KKT conditions are given by

$$
\begin{array}{cc}
N-\sum_{l=i}^{i+n-1} \frac{p_{i, l} N_{i}(l) e^{-\theta_{i} N_{i}(l)}}{1-p_{i, l}+p_{i, l} e^{-\theta_{i} N_{i}(l)}}-\mu_{i}=0 & \forall i \in \mathcal{A} \\
\frac{p_{i, l} \theta_{i} e^{-\theta_{i} N_{i}(l)}}{1-p_{i, l}+p_{i, l} e^{-\theta_{i} N_{i}(l)}}-\gamma_{l}+\lambda_{i, l}=0 & \forall i \in \mathcal{A}, l \in \mathbb{Z} \\
\gamma_{l}\left(\sum_{i \in \mathcal{A} \cap\{l-(n-1), \ldots, l\}} N_{i}(l)-N / R\right)=0 \quad \forall l \in \mathbb{Z} & \\
\lambda_{i, l} N_{i}(l)=0 & \forall i \in \mathcal{A}, l \in \mathbb{Z} \\
\mu_{i} \theta_{i}=0 & \forall i \in \mathcal{A}
\end{array}
$$

The equations (3) and (4) correspond to differentiating the Lagrangian with respect to respectively, $\theta_{i}$ and $N_{i}(l)$, while the remaining conditions are the complementary slackness conditions. Since the objective is convex in $\theta_{i}$ and $N_{i}(l)$, the KKT conditions are both sufficient and necessary conditions for optimality. From (4), we have $N_{i}(l)$ as a function of $\gamma_{l}, \theta_{i}$ given by

$$
N_{i}(l)=\max \left[0,-\frac{\eta_{i, l}}{\gamma_{l}} \log \frac{\eta_{i, l}}{\left(1-\eta_{i, l}\right)} \frac{\left(1-p_{i, l}\right)}{p_{i, l}}\right]
$$

where $\eta_{i, l}=\gamma_{l} / \theta_{i}$. From (3) and (5), we have that the parameters $\gamma_{l}, \theta_{i}$ are such that they satisfy

$$
\sum_{l=i}^{i+n-1} \eta_{i, l} \max \left[0,-\frac{\eta_{i, l}}{\gamma_{l}} \log \frac{\eta_{i, l}}{\left(1-\eta_{i, l}\right)} \frac{\left(1-p_{i, l}\right)}{p_{i, l}}\right]=N \forall i \in \mathcal{A}
$$

and

$$
\begin{gathered}
\sum_{i \in \mathcal{A} \cap\{l-(n-1), \ldots, l\}} R \max \left[0,-\frac{\eta_{i, l}}{\gamma_{l}} \log \frac{\eta_{i, l}}{\left(1-\eta_{i, l}\right)} \frac{\left(1-p_{i, l}\right)}{p_{i, l}}\right] \\
=N \quad \forall l \in \mathbb{Z}
\end{gathered}
$$

\section{DETERMINISTIC ARRIVALS}

We consider deterministic arrivals of data packets. In Subsections III-A, III-B and III-C, respectively, we consider the saturated (data packet every slot) case, the periodic (a data packet every $r$ slots) case and the general (data packets with an arbitrary repetitive pattern) case.

\section{A. Saturated arrivals}

Assuming that a new data packet arrives in every slot and under the stationarity assumption that $p_{i, l}=p_{i+n-l} \forall i, l$ equations (9) and (10) can be written as

$$
\begin{gathered}
\sum_{j=0}^{n-1} \eta_{i, i+j} \max \left[0,-\frac{\eta_{i, i+j}}{\gamma_{i+j}} \log \frac{\eta_{i, i+j}}{\left(1-\eta_{i, i+j}\right)} \frac{\left(1-p_{n-j}\right)}{p_{n-j}}\right]=N \\
\forall i \in \mathcal{A}
\end{gathered}
$$


and

$$
\sum_{j=0}^{n-1} R \max \left[0,-\frac{\eta_{l-j, l}}{\gamma_{l}} \log \frac{\eta_{l-j, l}}{\left(1-\eta_{l-j, l}\right)} \frac{\left(1-p_{n-j}\right)}{p_{n-j}}\right]=N
$$$$
\forall l \in \mathbb{Z}
$$

From inspection, a solution (which is unique due to the convexity of the optimisation) to the above set of equations is

$$
\eta_{i, l}=R
$$

and

$$
\gamma_{l}=\frac{R}{N} \sum_{j=1}^{n} \max \left[0,-R \log \frac{R}{(1-R)} \frac{\left(1-p_{j}\right)}{p_{j}}\right]
$$

For this solution to be non-trivial, $R<\max _{j=1, \ldots, n} p_{j}$ (to ensure $\gamma_{l}>0$ ). The optimal allocation is given by (8). From (11), it holds that $N_{i}(l)>0$ only if $p_{i+n-l}>R$. The following theorem provides a bound to the error probability $P_{e}$

Theorem 1. For saturated arrivals,

$$
P_{e} \leq e^{-\sum_{j \in[1, n]: p_{j}>R} R \log \frac{R}{p_{j}}+(1-R) \log \frac{1-R}{1-p_{j}}} .
$$

Proof: Each term of the sum log of the error probabilities in (1) is the same. Plugging the value of $\eta_{i, l}$ and $\gamma_{l}$ back into the objective (2) gives the bound to the error probability.

Note that for the erasure channel where $p_{j}=p$, the exponent boils down to the standard Kullback-Leibler divergence term $n D(R \| p)$. Note that $D(x \| y)$ denotes the divergence between the binary distributions $(x, 1-x)$ and $(y, 1-y)$ and is given by $x \log \frac{x}{y}+(1-x) \log \frac{1-x}{1-y}$. Furthermore, the optimal allocation is uniform, i.e., the contents of coded packet $C(i)$ are equally allocated among the $n$ transmitted packets in the interval $[i, i+n-1]$

\section{B. Periodic arrivals}

Consider now the case of periodic data packet arrivals where a new data packet arrives every $r$ 'th slot. Assume that the delay horizon $n$ is an integer multiple of $r$. Assuming the optimal $\theta_{i}=\theta$ and substituting $p_{i, l}=p_{i+n-l} \forall i, l$, equations (9) and (10) become

$$
\begin{gathered}
\sum_{j=0}^{n-1} \frac{\gamma_{i+j}}{\theta} \max \left[0,-\frac{1}{\theta} \log \frac{\gamma_{i+j} / \theta}{\left(1-\gamma_{i+j} / \theta\right)} \frac{\left(1-p_{n-j}\right)}{p_{n-j}}\right] \\
=N \forall i \in \mathcal{A}
\end{gathered}
$$

and

$$
\begin{gathered}
\sum_{j=q, r+q, 2 r+q, \ldots, n-r+q} R \max \left[0,-\frac{1}{\theta} \log \frac{\gamma_{i+q} / \theta}{\left(1-\gamma_{i+q} / \theta\right)}\right. \\
\left.. \frac{\left(1-p_{n-j}\right)}{p_{n-j}}\right]=N \quad \forall i \in \mathcal{A}, q \in\{0, . ., r-1\}
\end{gathered}
$$

for coded packet $l=i+q$ transmitted $q$ slots after the $i$ 'th information packet arrival with $q \in\{0, \ldots, r-1\}$. Clearly from (15), we see that the $\gamma_{l}$ values have a periodic structure with period $r$. Making use of this periodic structure we can rewrite
(13) as

$$
\begin{aligned}
\sum_{q=0}^{r-1} \frac{\gamma_{i+q}}{\theta} \sum_{j=q, r+q, 2 r+q, . ., n-r+q} \max \left[0,-\frac{1}{\theta} \log \frac{\gamma_{i+q} / \theta}{\left(1-\gamma_{i+q} / \theta\right)} .\right. \\
\left.. \frac{\left(1-p_{n-j}\right)}{p_{n-j}}\right]=N
\end{aligned}
$$

Substituting from (15), the above relation is just

$$
\sum_{q=0}^{r-1} \frac{\gamma_{i+q}}{\theta}=R
$$

So we are done if we can find $\theta$ and $\eta_{i+q}=\gamma_{i+q} / \theta, q \in$ $\{0, . ., r-1\}$ satisfying

$$
\begin{gathered}
\sum_{q=0}^{r-1} \eta_{i+q}=R \\
\sum_{j=q, r+q, 2 r+q, \ldots, n-r+q} \max \left[0,-\log \frac{\eta_{i+q}}{\left(1-\eta_{i+q}\right)} \frac{\left(1-p_{n-j}\right)}{p_{n-j}}\right] \\
=\theta N / R
\end{gathered}
$$

Note in the above, we have $r+1$ equations and $r+1$ unknowns. We do not have an analytic closed form expression for $\theta, \gamma_{q}$, nevertheless, we can numerically solve equations (16) and (17) and subsequently evaluate the bound to the error (see Section IV).

However, we do have closed form expressions for the erasure channel. It can be checked that if $R / r<p$, then the optimal $\eta_{i+q}=\frac{R}{r}$ and

$$
\theta=-\frac{n R}{N r} \log \frac{\frac{R}{r}}{\left(1-\frac{R}{r}\right)} \frac{(1-p)}{p} .
$$

Again by substituting back in the objective, we can bound the probability of error.

Theorem 2. For erasure channels and periodic arrivals,

$$
P_{e} \leq e^{-n D\left(\frac{R}{r} \| p\right)} .
$$

As in the saturated case, the optimal allocation is uniform over the $n$ length window of transmitted packets.

\section{General deterministic arrivals and the erasure channel}

The previous subsection considered the case when the arrival was once every $r$ slots. We now consider arbitrary arrival patterns which repeat after $r$ slots. We also assume $n$ to be a multiple of $r$. For arbitrary repetitive arrival patterns, the sum $\log$ utility is not equivalent to the error probability. (discussed in Section V). However, the two quantities are equivalent for the erasure channel. We will therefore focus on the erasure channel. In what follows, we will not use the determinism of the arrival pattern until the very last step. This is done to keep the formulation as general as possible. We therefore start off by assuming an arbitrary arrival pattern. 
Substituting $p_{k}=p \forall k$, equations (9) and (10) become

$$
\sum_{l=i}^{i+n-1} \eta_{i, l} \max \left[0,-\frac{\eta_{i, l}}{\gamma_{l}} \log \frac{\eta_{i, l}}{\left(1-\eta_{i, l}\right)} \frac{(1-p)}{p}\right]=N \forall i \in \mathcal{A}
$$

and

$$
\begin{gathered}
\sum_{i \in \mathcal{A} \cap\{l-(n-1), \ldots, l\}} R \max \left[0,-\frac{\eta_{i, l}}{\gamma_{l}} \log \frac{\eta_{i, l}}{\left(1-\eta_{i, l}\right)} \frac{(1-p)}{p}\right] \\
=N \forall l \in \mathbb{Z}
\end{gathered}
$$

We try for a solution with constant $\theta_{i}=\theta$. For a non-trivial solution we require $\eta_{i, l}<p$. Equation (19) is then

$$
-\frac{n_{l}}{\theta} \log \frac{\gamma_{l} / \theta}{\left(1-\gamma_{l} / \theta\right)} \frac{(1-p)}{p}=\frac{N}{R}
$$

where $n_{l}$ is the number of information packets arrived in the interval $[l-n+1, l]$. Solving (20) for $\gamma_{l}$,

$$
\gamma_{l}=\theta \frac{\frac{p}{1-p} e^{-\theta \frac{N}{R} \frac{1}{n_{l}}}}{1+\frac{p}{1-p} e^{-\theta \frac{N}{R} \frac{1}{n_{l}}}}
$$

It can be checked that $\eta_{i, l}<p$ is consistent with (21). Now substituting from (20) into (18) yields

$$
\sum_{l=i}^{i+n-1} \frac{\gamma_{l}}{n_{l}}=\theta R
$$

and substituting from (21) into (22),

$$
\sum_{l=i}^{i+n-1} \frac{1}{n_{l}} \frac{\frac{p}{1-p} e^{-\theta \frac{N}{R} \frac{1}{n_{l}}}}{1+\frac{p}{1-p} e^{-\theta \frac{N}{R} \frac{1}{n_{l}}}}=R
$$

If the above equation has a consistent solution for $\theta \forall l$, then we are done. For an arbitrary arrival pattern this will not be the case as the sum (23) involves the random variable $n_{l}$. We consider the case $R=1$ for simplicity, and choose $\theta$ to satisfy not (23), but (24) below:

$$
\sum_{l=i}^{i+n-1} \frac{1}{E\left(n_{l}\right)} \frac{1}{1+\frac{1-p}{p} e^{\frac{N \theta}{E\left(n_{l}\right)}}}=1
$$

The relation (24) is obtained from (23) by replacing $n_{l}$ with its expected value and, since $E\left(n_{l}\right)=\lambda n$, simplifies to yield

$$
\theta=\frac{-n \lambda}{N} \log \left(\frac{\lambda(1-p)}{p(1-\lambda)}\right)
$$

We now substitute the guess for $\theta$ (given by 25) and for $N_{i}(l)$ given by $N_{i}(l)=N / n_{l}$ into the objective (2). We have

$$
\begin{aligned}
& \sum_{i \in \mathcal{A}}\left(\theta N+\sum_{l=i}^{i+n-1} \log \left(1-p+p e^{-\theta N_{i}(l)}\right)\right) \\
& =\sum_{i \in \mathcal{A}}\left(\theta N+\sum_{l=i}^{i+n-1} \log \left(\frac{p \theta e^{-\theta N_{i}(l)}}{\gamma_{l}}\right)\right)
\end{aligned}
$$

$$
\begin{aligned}
& =\sum_{i \in \mathcal{A}}\left(\theta N+\sum_{l=i}^{i+n-1}\left(\log \left(\frac{p \theta}{\gamma_{l}}\right)-\theta N_{i}(l)\right)\right) \\
& \stackrel{(a)}{=} \sum_{i \in \mathcal{A}} \sum_{l=i}^{i+n-1} \log \left(\frac{p \theta}{\gamma_{l}}\right)-\sum_{i \in \mathcal{A}^{C}} \theta N+\sum_{l: n_{l}=0} \theta N \\
& \stackrel{(b)}{=} \sum_{i \in \mathcal{A}} \sum_{l=i}^{i+n-1} \log \left(\frac{p\left(1+\frac{p}{1-p} e^{-N \theta / n_{l}}\right)}{\frac{p}{1-p} e^{-N \theta / n_{l}}}\right)-\sum_{i \in \mathcal{A}^{C}} \theta N \\
& +\sum_{l: n_{l}=0} \theta N \\
& =\sum_{i \in \mathcal{A}} \sum_{l=i}^{i+n-1} \log \left(p+(1-p) e^{N \theta / n_{l}}\right)-\sum_{i \in \mathcal{A}^{C}} \theta N+\sum_{l: n_{l}=0} \theta N \\
& \stackrel{(c)}{=} \sum_{i \in \mathcal{A}} \sum_{l=i}^{i+n-1} \log \left(p+(1-p)\left(\frac{\lambda(1-p)}{p(1-\lambda)}\right)^{-n \lambda / n_{l}}\right)-\sum_{i \in \mathcal{A}^{C}} \theta N \\
& +\sum_{l: n_{l}=0} \theta N \\
& =\sum_{l} n_{l} \log \left(p+(1-p)\left(\frac{\lambda(1-p)}{p(1-\lambda)}\right)^{-n \lambda / n_{l}}\right)-\sum_{i \in \mathcal{A}^{C}} \theta N \\
& +\sum_{l: n_{l}=0} \theta N
\end{aligned}
$$

where the equality (a) follows from the observation that

$$
\sum_{i \in \mathcal{A}} \sum_{l=i}^{i+n-1} N_{i}(l)=\sum_{l: n_{l} \neq 0} N
$$

the equality (b) follows from substitution for $\theta / \gamma_{l}$ using (21) and the equality (c) follows from guess for $\theta$ using (25).

We therefore have the following bound on the sum log of the error probabilities:

$$
\begin{aligned}
& \sum_{i} \log \operatorname{Pr}\left(\sum_{l=i}^{i+n} N_{i}(l) \delta_{i, l}<N\right) \leq \sum_{l} n_{l} . \\
& . \log \left(p+(1-p)\left(\frac{\lambda(1-p)}{p(1-\lambda)}\right)^{-n \lambda / n_{l}}\right)-\sum_{i \in \mathcal{A}^{C}} \theta N+\sum_{l: n_{l}=0} \theta N
\end{aligned}
$$

which holds for any arbitrary (not necessarily repetitive) arrival pattern.

Repetitive arrivals - If the arrival pattern has period $r$ and if $n$ is a multiple of $r$, then $n_{l}=n \lambda$, where $\lambda$ is the arrival rate of packets. We plug this value in (26) to get

$$
\begin{aligned}
\sum_{i \in \mathcal{A}} \log \operatorname{Pr} & \left(\sum_{l=i}^{i+n} N_{i}(l) \delta_{i, l}<N\right) \\
& \leq \sum_{l} n \lambda \log \frac{p}{\lambda}-\sum_{i \in \mathcal{A}^{C}} \theta N+\sum_{l: n_{l}=0} \theta N
\end{aligned}
$$

Normalizing all the terms by the total number of arrivals $|\mathcal{A}|$, observing that $|\mathcal{A}|=T \lambda$, and that $n_{l} \neq 0$ for $n$ which is a 
multiple of $r$, we have

$$
\begin{aligned}
\frac{1}{|\mathcal{A}|} \sum_{i \in \mathcal{A}} \log \operatorname{Pr} & \left(\sum_{l=i}^{i+n} N_{i}(l) \delta_{i, l}<N\right) \\
& \leq n \log \frac{p}{\lambda}+n(1-\lambda) \log \frac{\lambda(1-p)}{p(1-\lambda)} \\
& =-n D(\lambda|| p) .
\end{aligned}
$$

As $N_{i}(l)$ is uniform, each term inside the summation of the L.H.S in (27) is equal to the R.H.S. In other words, for any packet, the probability of the packet not being decoded is upperbound by the exponential decay in $n$. We thus have the following theorem:

Theorem 3. For general deterministic arrivals and for an erasure channel, the error probability $P_{e}$ is bounded by

$$
P_{e} \leq e^{-n D(\lambda \| p)} .
$$

\section{NUMERICAL RESUlts}

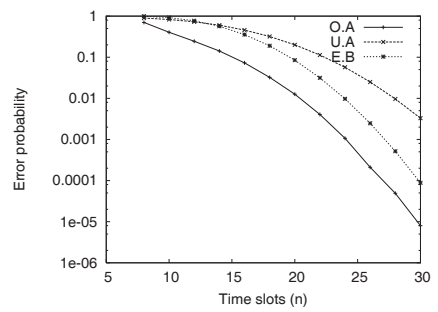

Fig. 3. $\lambda=0.5, p_{i, l}=1-0.9^{i+n-l}$. O.A and U.A denote the error plots under, respectively, optimal and uniform allocation, and E.B denotes the error bound under optimal allocation

We ran simulations for the case when the channel probabilities are given by $p_{i, l}=1-0.9^{i+n-l}$ ( alternately $p_{j}=1-0.9^{j}$ ) and the arrival rate is one packet every two time slots, i.e., when $\lambda=0.5$. The Figure 3 plots the error probability versus the decoding deadline for the optimal allocation that minimizes the bound (2) ( obtained from numerically solving (16) and (17)) and also plots the corresponding bound to the error probability given by (2). The performance of the uniform allocation (optimal for an erasure channel) is also compared to the (w.r.t objective (2)) optimal allocation and is seen to be significantly worse than this optimal allocation.

The Figure (4) compares our two stage coding scheme with a causal Fountain code (which sends a linear combination of undecoded packets at the receiver each slot) over the erasure channel assuming infinite field and no decoding overhead. The allocation which minimizes the objective (2) is uniform and and we therefore refer to our scheme as Uniform coding with no Feedback (see Fig. 4). We numerically compare our scheme with the causal Fountain coding scheme and observe only a slightly worse performance for our scheme. It is however unfair to make a direct comparison since the causal coding scheme requires feedback to limit the queue size at the sender.

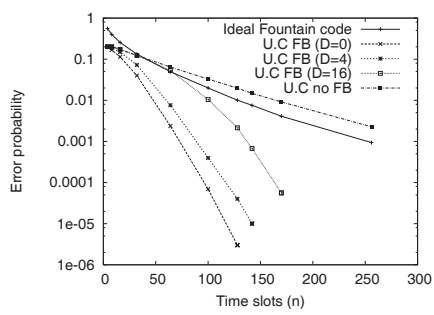

Fig. 4. $\lambda=0.25, p=0.33$ U.C FB denotes uniform allocation with feedback. $D$ is the delay (in slots) of feedback.

If we incorporate feedback into our scheme, we observe a dramatic reduction in error probabilities. The incorporation of feedback is explained as follows: Without feedback the allocation for each data packet (arrived in the past $n$ timeslots) in a transmitted packet is $N / n_{l}=N / n \lambda$. With feedback, the sender drops the data packets which have been decoded at the receiver and thus the new allocation is $N / \breve{n_{l}}$ where $\breve{n_{l}}$ is $n_{l}$ less the number of packets decoded at the receiver. Fig. 4 shows the error probability curves for different amount of delays in the feedback. The numerical studies make a strong case for our coding scheme, especially in the light of dramatically improved performance using feedback.

\section{SUM LOG UTILITY AND ERROR PROBABILITY}

In all the cases considered so far, the sum log of the error probabilities provided a direct handle on the error probabilities themselves. For general reordering channels and arrival patterns, the two quantities are not equivalent. Consider the indicator of the error event for the packet arrived at the $i^{\text {th }}$ slot given by $Z_{i} \triangleq 1_{\left\{\sum_{l=i}^{i+n-1} N_{i}(l) \delta_{i, l}<N\right\}}$. The normalized sum $\log$ of the error probabilities is given by $\frac{1}{|\mathcal{A}|} \sum_{i \in \mathcal{A}} \log \mathbb{E}\left[Z_{i}\right]$ where the expectation is over the channel randomness. Note, $Z_{i}$ is not identically distributed. However, it holds that $Z_{i}$ is i.i.d modulo $n$, i.e., the sequence $\left\{Z_{l n+q}\right\}_{l \geq 0}$ is i.i.d for $q=0, \ldots, n-1$. It therefore holds that

$$
\frac{1}{|\mathcal{A}|} \sum_{i \in \mathcal{A}} \log \mathbb{E}\left[Z_{i}\right]=\frac{1}{n \lambda} \sum_{i \in\{[0, n-1]\} \cap \mathcal{A}} \log \mathbb{E}\left[Z_{i}\right] .
$$

On the other hand, the error probability is given by

$$
\lim _{|\mathcal{A}| \rightarrow \infty} \frac{1}{|\mathcal{A}|} \sum_{i \in \mathcal{A}} Z_{i}=\frac{1}{n \lambda} \sum_{i \in\{[0, n-1]\} \cap \mathcal{A}} \mathbb{E}\left[Z_{i}\right] .
$$

Thus the exponent of the error probability is given by $\log \frac{1}{n \lambda} \sum_{i \in\{[0, n-1]\} \cap \mathcal{A}} \mathbb{E}\left[Z_{i}\right]$ and by Jensen's inequality, this exponent upperbounds the exponent in (28). Note that for the erasure channel, both exponents are equal as the $Z_{i}$ 's are identically distributed (though not independent).

\section{REFERENCES}

[1] E. Martinian, C-E. Sundberg "Burst erasure correction codes with low decoding delay" , IEEE Transactions on Information theory, vol. 10, pp.2494-2502, 2004.

[2] A. Sahai, "Any-time information theory", Ph.D Dissertation, 2001. 